2 Neusser: Wiemer klinische Wochenschrift, 1894, No. 39 3 Kolisch: Wiener tklinische Wochenselnrift, 189i, p. 787 i Rachford: Medical News, Nov. 3 , 189

5 Schmoll: Zestochr. f. klinische Medecin., 1896, Bd. xxix, p. 510

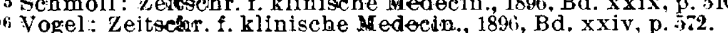

\section{THE OCULAR EXPRESSIONS OF GOUT.}

Fresented in the Section on Practice of Medicine at the Forty eighth Annual Meeting of the American Medical Association held at Philadelphia, June 1-4, 1897.

BT CHARLES A. OLIVER, A.M., M.D.

OSE OF THE ATTENDING SLRGEONS TO WILLS' EYE HOSPITAI; ONE OF TH OPHTHALMTC SURGFONS TO THE PHILADEJPHIA HOSPITAL, ETC. PHILATELPHIA, PA.

When it is remembered that the visual bulb has within its structures a numerous network of blood vessels, and when it is understood that the eyeball is nothing more than a lymph-holding end organ which is constantly changing the greater part of its fluid constituents, it can be realized that in these structures there can appear all of the irritation and inflammatory ehanges that may be produced by either an undue amount of urates in the system, or the existence of uric acid and other abnormal products in the adjacont parts.

The presence of material in the outer coats of the eye, that is so similar to that found in any jointtissue, and the close relationship that the bulbus itself in the orbit, bears to every ball-and-socket joint. both show how the external coverings of the organ, its encompassing capsules, and its attached muscles with their delicate sheaths can be seriously disturbed by fluid-change, inflammatory exudate, and inorganic deposit, as the result of a gouty diathesis.

The accessories too, so abundant in gland, vessel, lymph-channel, cartilage and periosteum, furnish an abundant soil for toxic reaction, abnormal accretion, and arthritic change. Nor do the associated nerves escape. Here the evil accompaniments of pressure, invasion, inflammation and degeneration, all exhibit themselves in many types of the disorder.

Take for example the lids, the conjunctiva, the lachrymal apparatus and the orbital contents. How characteristic are the swollen, tumid and angry lids, so excruciatingly painful at times, and so seldom, if ever, ulcerated! The inflammatory condition so rapid on onset, so barometric in character, and so quickly subsiding by the application of dry heat and the employment of constitutional measures, makes a symptomatic grouping that once seen is seldom, if ever, forgotten. The fleeting forms of conjunctival inflammation with slight or no excretion, the sensation of the presence of foreign body, the dense and not infrequent edema of the sub-mucous tissue, and the intense intolerance to light, must all be noted as expressions of the general disorder, and not believed to be entirely the consequences of colonization of local. ized bacteria. Lithiasis conjunctivæ or calcified infarcta occurring in the course of the Meibomian glands, are frequently seen in middle-aged and elderly subjects, and especially in men, as evidence of similar changes to be found elsewhere. At first causing no disturbance, but later, by perforation and exposure, producing all manner of irritative lesions by mechanical action, they may produce that most dangerous complication, desquamation of the corneal epithelium, with the usual train of infectious sequelæ. Keratitis, by some stated, peculiarly band-like in character, interstitial at times, and rarely, if ever, ulcerous in form, has been spoken of by a number of competent authorities.
Inflammation of the sclera both in the fibrous intima and in its overlying capsular and tendinous material, evidences, particularly in the male subject, an intimate relation to the general condition by a series of extremely fugitive signs that are in many cases pathognomonic. Circumscribed when super. ficial, it is not rarely associated with such intense dread of light and such sharp and frequently recurring attacks of lancinating pain, amidst copious gushes of hot tears, that the condition of the sufferer becomes truly pitiable. As suddenly disappearing by the local use of heat with pilocarpin at times, and the internal administration of large doses of alkalies, the comfort obtained is ofttimes phenomenal and truly appreciated.

So too with the deeper structures of the eyeball. The iris with its ciliary and choroidal prolongations may suffer intensely. Frequently sharp, excessively painful, devoid of much gross alteration, and at times provoked by accidental and operative traumatism, the anterior segment of the uveal tract becomes a treacherous ground for more deeply seated and more disastrous future outbreaks. More often the inflammation, which may be either plastic or serous in type, especially the latter, is marked and insidious. At times it is hereditary, with vitreous complications. As a rule it is associated with the general exacerbations and is untypical in form, and although, probably in many cases, especially in the serous variety, it is coextensive with some more deeply seated inflammatory change, there is very little by which it can be separated etiologically from any other similar form of inflammatory reaction.

The local treatment in both of these types of disorder (always employing heat for the relief of pain), is dependent upon the coexxistent condition of the organ, taking care if any permanent success be hoped for, to resort to active and properly applied systemic measures.

Should the middle or the posterior thirds of the uveal tract be involved; that is, should the ciliary body with its contained muscles, and the choroid be inflamed, which fortunately is not so frequently the case, the diagnosis becomes less easy, the evil consequences are greater, and the treatment is less availing. It is probable that in this peculiar type of disorder, the existence of most of the forms of lenticular change and some of the varieties of glaucomatic process must be sought for.

Where the retina and optic nerve are concerned, the ophthalmoscopic picture becomes in many cases quite typical. Rapidly recurrent fine feathery hemorrhages with more deeply seated ones located throughout the retina and atonic broadening and flattening of the retinal veins and arteries, associated at times with some of the signs of incipient glaucoma, often appear early in the history of the case. Later, in the more chronic forms, inflammatory vessel-wall change, with shrinkage, atheroma and sclerosis, first manifesting itself in the veins and subsequently in the arteries, may be found. The easily recognized edema of the retina with its usual venous accompaniments generally confined to the posterior pole of the eye, followed by insidious though progressive inflammatory reaction extending both anteriorly by contiguity into the vitreous and the lens, and posteriorly into the choroid are now superadded. These, with the at times, peculiar glistening yellowish bodies found aggregated more particularly in the superficial layers 
of the circum-macular region, may be considered as almost pathognomonic. Often low grades of neuritis which seemingly extend some distance back into the nerve-substance can be recognized, these ordinarily appearing monolaterally and generally found in middle aged subjects.

As a rule, uncontemporaneously binocular, generally first appearing in the visual field, and associated with defective vision; the retinal and optic nervegroupings are not difficult of recognition. Prognosis is never good and incomplete blindness is almost certain.

From this clinical study, it will be seen, as before hinted, that the eyeball, with its accessories, is peculiarly prone to disturbance during the course of general disease; in fact, the organ in its every detail of structure, is liable to changes that are dependent upon gout.

\section{GASTRO-INTESTINAL AND HEPATIC RELATIONS OF GOUT.}

Presented in the Section on Practice of Medicine at the Forty-eighth Annual Meeting of the American Medical Association, beld at Philadelphia, Pa., June 1-4. 1897.

BY CHARLES G. STOCKTON, M.D.

Professor of the Principles and Practice of Medicine and Clinical Yfedicine, Medical Department, University of Buffalo; Attending

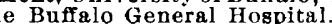
BUFFALO, N. Y

Murchison and Sir Dyce Duckworth, among other students of the subject, have associated the excessive formation of uric acid and the development of gouty attacks with disturbances of the liver. Under the name of lithemia or latent gout, Murchinson described a set of symptoms very commonly met with in this country; among the conspicuous symptoms is the appearance of uric acid, urates and calcium oxalate crystals in the urine in abnormal amount.

These cases of lithemia show evidence of disturbed primary digestion, congestion of the liver, headache, lassitude, malaise, but rarely evidences of deposits, arthritic or otherwise, that are characteristic of true gout.

The question has been raised, and I think justly, is lithemia gout or is it the expression of a toxemia resulting from habitual disorder of the digestive organs including the liver? Before answering the question it may be well to turn to some later views regarding the relation of uric acid to gout, and the origin of uric acid in general.

It would appear to have been conclusively shown by Sir Alfred Garrod, Sir William Roberts, and later by Arthur P. Luff in his recent Goulstonian lectures, first, that "uric acid is not normally present in the blood of man or other mammals nor in the blood of birds; second, that uric acid is normally produced in the kidneys only, and is formed from urea, probably by the conjunction of that substance with the glycocin in the kidneys."

Since the glycocin has its origin in the liver, we can readily understand how hepatic disorder might lead to disturbances in the amount and quality of the glycocin formed. We may therefore understand how hepatic diseases, either with or without gout, may be competent to derange urinary secretions. So it will be seen that an excess of uric acid in the urine does not necessarily mean a gouty diathesis. In fact, it has been shown by Pfeiffer that the uric acid output, instead of being increased in gout, is in point of fact diminished, and the kidneys seem to have lost in part their power of elimination; and although we find in he blood of the gouty individual uric acid in the form of quadurates, and also find the deposits in the joints and other parts not in the form of uric acid, but in that of the biurate of soda, this takes place not because there is uric acid in the blood, but because of some reason that we do not yet understand.

This proposition, namely, that gouty deposits do not follow merely because there is uric acid in the blood, may be proven by the fact that in leukemia and several other affections in which there is rapid growth and destruction of leucocyles, there is formed a relatively enormous amount of uric acid from nucleinic acid, and yet in these cases we find none of the ordinary symptoms of gout, no deposits of the biurate of soda in the tissues, but we do find the uric acid passing from the body, partly unchanged and partly in the form of urea.

It will thus be seen, 1, that gout does not depend upon the continued presence of the excess of uric acid in the blood; 2 , that the formation of uric acid in the kidneys is disturbed in case of gout, probably as a result of some disorder of the liver; 3 , that disorders of the liver unaccompanied by gout are capable of deranging the normal uric acid output.

Let us now return to the question already propounded. Is lithemia gout, or is it the expression of a toxemia resulting from habitual disorder of the digestive organs, including the liver? From experi. ence gained in the study of functional disorders of the stomach the conclusion has been forced upon me that the great majority of instances of so-called lith. emia are in fact cases of toxemia, in no true sense gouty in nature. As there are many causes of functional disturbance in digestion, so there are many causes of lithemia. It is readily admitted that gout is the occasional cause of the disorder of primary digestion, but the proportion of cases in which it is shown as an actual probability is extremely small.

Perhaps the full import of this position will be more clearly shown when it is pointed out that permanent relief of the condition does not follow the treatment directed toward gout, but that it does follow the right ordering of diet and the right management of the disturbed digestion in other ways. Indoubtedly it is true that digestive diseuses aggravate gout, and I think that Luff has shown why it is that indiscretion in diet, particularly as regards certain kinds of food, are capable of exciting paroxysms of gout in gouty subjects. I have carefully and repeatedly studied the stomach contents in a few cases of gout, and find that quite uniformly there occur periods of marked hyperchlorhydria with delayed starch digestion and flatulence, followed by enlargement of the liver, which conditions, if not relieved, are likely to be succeeded by characteristic arthritic attacks.

At other times such indiscretions in gouty subjects excite paroxysms of gastralgia or angina pectoris, the latter usually accompanied by high arterial tension, and sometimes by the discharge of a large amount of pale-colored urine. Again, an acute eczema makes its appearance. I have found that the hyperchlorhydria occurring in the gouty is very intractable to treatment, and those remedies (acting through the nervous system) that generally prove useful in hyperchlorhydria depending upon reflex nervous causes, in gouty patients are of little value. Large and repeated doses of alkalies and potassium iodid give the best result.

Now, such patients may not observe a strict dietary 